Der folgende Artikel legt Zeugnis dafür ab, dass sich die Hausärzteschaft, die Institute für Hausarztmedizin und Berufsverbände im Bereich Palliativmedizin engagieren. Diesem Engagement kommt gerade in Zeiten der COVID-19-Pandemie eine besondere Bedeutung zu. Bleibt zu hoffen, dass sich die Rahmenbedingungen noch deutlich verbessern, damit eine Palliativmedizin, wie sie angedacht ist, auch umgesetzt werden kann. Am Einsatz der Medizinal- und Gesundheitsberufe liegt es offensichtlich nicht.

\title{
Palliative Care und Hausarztmedizin
}

\author{
Christoph Cina ${ }^{a}$, Andreas Lischera, Christoph Merloa, Christian Studera, Linda Hadorn ${ }^{b}$ \\ a Fachgruppe Ärzte palliative ch, Institut für Hausarztmedizin \& Community Care Luzern (IHAM \& CC); ${ }^{b}$ Abteilung Public Health FMH
}

\section{Vom Commitment zur konkreten Umsetzung}

Grosse Grundversorgergesellschaften wie SGAIM und mfe anerkennen zusammen mit palliative ch, dass palliative Patientinnen und Patienten regional und in einem interprofessionellen Rahmen betreut werden sollten. Sie setzen sich dafür ein, dass unter ihren Mitgliedern die Standards und Werkzeuge der Palliative Care (wie runder Tisch, Betreuungsplan und Assessment wie beispielsweise nach SENS-Modell [1]) bekannt sind und der erhöhte Arbeitsaufwand, der bei palliativen Patienten notwendig ist, im Tarif korrekt abgebildet wird [2]. Eine erfolgreiche interprofessionelle Zusammenarbeit richtet sich nach der unantastbaren Würde des Menschen. Die Erkenntnis, dass der Mensch in seiner Vulnerabilität in der letzten Lebensphase auf Hilfe angewiesen ist und nicht nur ein Anrecht auf ein würdevolles Leben, sondern auch Sterben hat, soll Anlass einer neuen Sorgekultur im Sinne einer Caring Community sein. Diese Kultur setzt eine interprofessionelle Zusammenarbeit zum Wohle des "umsorgten" Patienten voraus, im Sinne einer Medizin, bei der die Patienten im Zentrum stehen. Man könnte auch von einer kommunalen Sorgekultur sprechen mit Vernetzung der verschiedenen «Welten», welche sich verantwortungsvoll und vertrauensvoll beteiligen.

\section{Hausarztmedizin in einer Caring Community}

Der Hausarzt betreut seine Patienten über Jahre und ist in der Regel ihr erster Ansprechpartner in Gesundheitsfragen. Er kommt auch nach Hause, kennt die lokalen Verhältnisse und verfügt über ein grosses Beziehungsnetz. Dieses Berufsverständnis gibt dem Hausarzt einen unersetzlichen Mehrwert, den er in die interprofessionelle Zusammenarbeit einbringen kann.

\section{Voraussetzungen für den Erfolg einer interprofessionellen Zusammenarbeit}

Gemeinsame Werte prägen eine fruchtbare Zusammenarbeit wie die Bereitschaft, die Bereiche der verschiedenen Professionen mit ihren Kompetenzen und Fähigkeiten kennenzulernen und sie als Bereicherung zu erfahren und nicht als Konkurrenz. Das Arbeitsverhältnis zeichnet sich durch eine gegenseitige Wertschätzung aus, ein Arbeiten «auf Augenhöhe» mit synchronisierten Arbeitsabläufen und Handlungen. Zentral ist dabei die Zielsetzung, sich nach den Bedürfnissen, Symptomen und Problemstellungen der betroffenen Patientinnen und Patienten sowie ihrer nahestehenden Bezugspersonen zu richten. In der konkreten Betreuung von Patienten gilt es, am sogenannten «runden Tisch» die Kompetenzen, Fähigkeiten und Verantwortlichkeiten zu klären. Als Basis einer erfolgreichen Zusammenarbeit dienen dabei konsensbasierte Standards und Arbeitswerkzeuge.

\section{Konsensbasierte Standards und Tools}

Das SENS-Modell hat sich in der Grundversorgung als mögliches Assessmentinstrument zunehmend verbreitet [1]. Mit den Kompetenzen «Entscheidungsfindung» und "Symptom-Management» wird die Autonomie des Menschen mit dem Ziel der Selbstbestimmung und Selbsthilfe unterstützt. Die Kompetenzen «Netzwerkorganisation» und "Support der Bezugspersonen» fördern die soziale Integrität und bieten Sicherheit im Sinne von "Aufgehobensein».

Die erwähnten Kompetenzen im SENS-Modell lassen sich modular in einem Betreuungsplan [3] abbilden. Dieser dient als Handlungsrichtlinie des interprofessionellen Teams. Damit sollen die Grundbedürfnisse unseres menschlichen Daseins respektiert und die Würde des Menschen gewährt sein.

Polymorbide Patientinnen und Patienten werden nicht selten durch eine ausgedehnte Polypharmazie 
belastet. Ein einheitlicher Medikamentenplan, bei dem alle nicht indizierten Medikamente abgesetzt werden, verbessert einerseits die Medikamentensicherheit und reduziert andererseits die Behandlungslast des Patienten. Dabei kann eine digitale interprofessionelle Plattform [3], bei der alle beteiligten Professionen beteiligt sind, die Kommunikation stark verbessern.

\section{Hohe Zahl an Hausbesuchen}

Gemäss einer aktuellen, schweizweiten Erhebung im Sentinella-Netzwerk (noch nicht publizierte Daten IHAM \& CC) bieten $84 \%$ der befragten Hausärzte Hausbesuche an, welche $1,8 \%$ aller Konsultationen ausmachen. 50\% der Hausbesuche finden in Institutionen

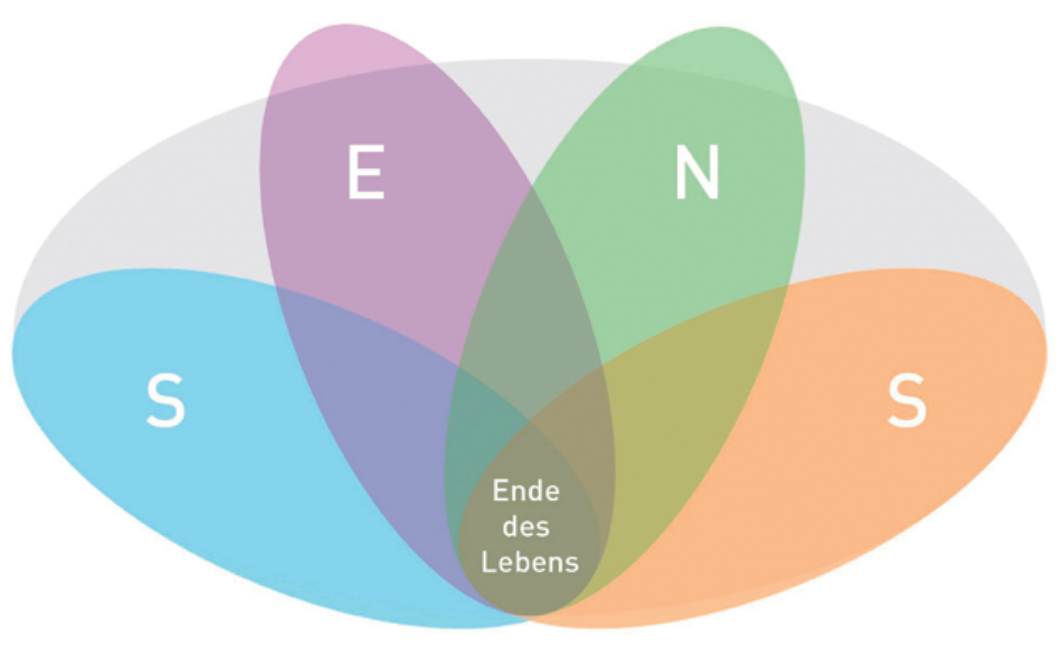

\section{Grundlagen}

Transversale Kompetenzen, die sowohl für das Symptom-Management, die Entscheidungsfindung. das Netzwerk und den Support als Grundvoraussetzungen gelten damit Palliative Care/die Palliativversorgung erfolgreich umgesetzt werden kann.

\section{Symptom-Management}

Beste Möglichkeiten der Symptombehandlung und Empowerment zur Selbsthilfe in der Symptombehandlung.

\section{Entscheidungsfindung}

Definition der eigenen Ziele und Prioritäten, schrittweise, selbstgesteuerte Entscheidungsfindung und präventive Planung für mögliche Komplikationen.

\section{Ende des Lebens}

Hat seine eigenen Herausforderungen, die sowohl das Symptom-Management, die Entscheidungsfindung. das Netzwerk und den Support betreffen. Die Entscheidungsfindung wird jedoch meist als am schwerwiegendsten empfunden.

Netzwerk

Aufbau eines Betreuungsnetzes unter Zusammenführung von ambulanten (Betreuung zuhause) und stationären Strukturen.

\section{Support}

Aufbau von Unterstützungssystemen für die Angehörigen, auch über den Tod hinaus: Unterstützungmöglichkeit auch für die beteiligten Fachpersonen.

\section{Ein neues CAS Palliative Care in Luzern}

Seit Herbst 2019 bietet die Universität Luzern in Kooperation mit dem Kantonsspital Luzern einen neuen Zertifikatslehrgang "CAS Palliative Care" als fokussierte Weiterbildung an. Als Teil des ersten Moduls "Palliative Medizin und Begleitung" ist ein Kurstag dem Thema "Palliative Care in der Hausarztmedizin und HomeBased Palliative Care» gewidmet. Nach einem mehrstündigen interaktiven Referat mit facettenreicher Einführung ins Thema (Christoph Cina) führt das Institut für Hausarztmedizin \& Community Care Luzern am Nachmittag drei parallele Workshops durch.

wie Pflegeheimen statt. Die meisten Befragten Ärztinnen und Ärzte erachten Hausbesuche als wichtig oder sehr wichtig. Als häufigste Motivation für Hausbesuche wird die Serviceleistung für den Patienten genannt.

\section{Workshops im Rahmen des CAS Palliative Care in Luzern}

In den folgenden Workshops werden verschiedene Schwerpunkte der Palliative Care in der Hausarztmedizin anhand von Patientenbeispielen beleuchtet.

\section{«Symptomkontrolle im hausärztlichen Setting» (Andreas Lischer)}

Das erste «S» im SENS-Assessment betrifft die Symptome und widerspiegelt oft auch die «erste» Sorge unserer Patienten, wenn es um ihre letzte Lebensphase geht: «Muss ich ersticken?», «Werde ich starke Schmerzen haben?», «Werde ich verwirrt sein?». Ein durchdachter und verständlicher Medikamenten-Notfallplan, aus dem fundierte Kenntnisse der aktuellen Situation und vorausschauende Planung ersichtlich sind, kann diesen Fragen die Schärfe nehmen und Vertrauen schaffen. Unabdingbar ist, dass dieser Plan auch in der Nacht und am Wochenende funktionieren muss, wenn unerwünschte und unnötige Hospitalisationen vermieden werden sollen.

Bei sich rasch verändernder Situation sind allerdings zusätzlich die mentale und physische Präsenz des Hausarztes sowie seine telefonische Erreichbarkeit gefragt. Ein paar Hilfsmittel in der Hand des Hausarztes können hilfreich sein: Das Pulsoxymeter oder ein Präsenzlabor erleichtern in bestimmten Situationen eine Entscheidungs- oder Dosisfindung. Elektronische Tools unterstützen ihn zum Beispiel bei der Opioid-Rotation oder bei Fragen der Zermörserbarkeit von Tabletten. Meist genügen aber menschliches Gespür sowie klinische Intuition und Professionalität für eine tragfähige Entscheidung. 
«Würde und Demenz - eine besondere Herausforderung" (Christian Studer)

Der Anteil an über 85-jährigen demenzkranken Menschen liegt bereits im zweistelligen Prozentbereich. Nicht selten zieht sich die Palliative Care über Monate bis Jahre hin. Die Urteilsfähigkeit nimmt sukzessive ab. Bei Vorliegen einer dementiellen Entwicklung hat die schweizerische Rechtsprechung eine Beweislastumkehr entschieden: Wer Urteilsfähigkeit behauptet, trägt dafür Beweislast [4]. Eine Teil-Urteilsfähigkeit für spezifische Fragestellungen muss also bewiesen werden.

Bei der Betreuung stehen aber nicht die Defizite, sondern die Ressourcen im Vordergrund, um die Restautonomie, Sicherheit und das Selbstwertgefühl der Demenzerkrankten und somit deren Würde zu fördern. Es gilt, diese Restautonomie möglichst lange zu erhalten und dabei die aktuellen Aktivitätsmöglichkeiten des Patienten zu evaluieren und zu respektieren. Da Brücken in die Vergangenheit einbrechen, können auch neue Werte entstehen, die die Umgebung brüskieren können. Es braucht eine Grosszügigkeit der im Umfeld betreuenden Menschen mit dem Augenmerk auf die Balance zwischen Erhalt einer Teil-Autonomie und Übernahme von Fürsorge.

\section{"Chancen und Risiken der Palliativbetreuung - mit den Angehörigen im gleichen Boot» (Christoph Merlo)}

Interessanterweise betrachten sich sowohl die Angehörigen als auch die medizinischen Fachkräfte wie Ärzteschaft und Pflegende als Schlüsselpersonen für die Koordination der häuslichen Palliativbetreuung [5].
Eine Rollenklärung und gegenseitige Unterstützung sind deshalb Voraussetzung, auch um anhand gezeigter Beispiele bei Situationen mit schwer kontrollierbaren Schmerzen, einer Stuhl- und Urininkontinenz oder exulzerierenden Tumoren eine gute Betreuung zu erreichen. Auf die persönlichen und sozialen Ressourcen der Angehörigen ist zu achten, gemäss deren die betreuenden Pflegefachkräfte, der Hausarzt und weitere Personen des Betreuungsteams Unterstützung gewährleisten.

\section{Wie schaffen wir die konkrete Umsetzung?}

Palliative-Care-Angebote sollten zum festen Bestandteil von Ausbildung der künftigen Grundversorger und der Fortbildung werden. Das Lernen aus positiven Beispielen (Modelle guter Praxis) eignet sich insbesondere auch für hausärztliche, interdisziplinäre und interprofessionelle Qualitätszirkel. Idealerweise können so in der praktischen Versorgung eigentliche Care Communitys gebildet werden.

Literatur

1 Eychmüller Steffen. SENS macht Sinn - Der Weg zu einer neuen Assessment-Struktur in der Palliative Care. Ther Umsch. 2012 Feb;69(2):87-90.

2 Sind wir Hausärzte bereit für Palliative Care? PHC. 2020;20(2):77-8.

3 https://www.palliative-so.ch/Angebote/Arbeitsinstrumente-fuerFachpersonen/PKuEl/

4 https://www.unilu.ch/fileadmin/fakultaeten/rf/aebi/dok/Jusletter_Urteilsunfaehiger_Patient.pdf

5 Fachhochschule Nordwestschweiz, Hochschule für Angewandte Psychologie. Projektbericht «Palliative Care in der Schweiz - die Sicht der Leistungserbringenden" (NRP 74).

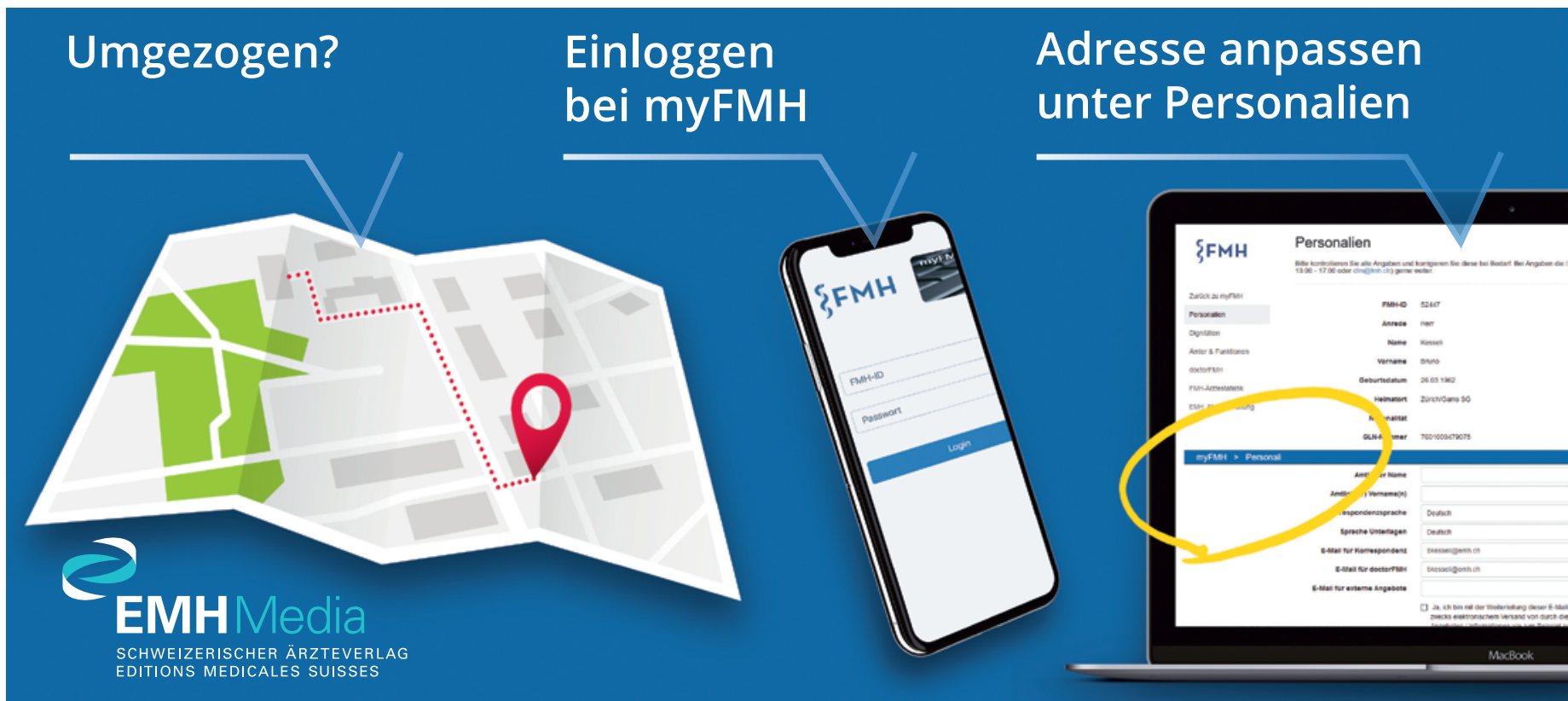

